\title{
WHAT CAN WE LEARN FROM Li AND BE STELLAR OBSERVATIONS?
}

\section{H. REEVES}

I want to state a few questions which are raised by the observations of stellar $\mathrm{Li}$ and $\mathrm{Be}$, and to see how far we can go in giving answers.

I do not believe that we can, at the present time, give very definite answers to most of these questions and build up a unique model in which all observations are properly understood. We are still faced with different possibilities which can not be excluded, but can only be classified according to their degree of 'plausibility'. Although we are led to choose one of these possibilities in the light of present data, it seems wise to keep the other possibilities in the back of our mind, and to assess their merit. Considering this, a few alternate models will be described and discussed.

A. Were the $\mathrm{L}$ elements $\mathrm{Li}, \mathrm{Be}, \mathrm{B}$ generated in the star in which they are observed?

The following observations suggest that at best, a small fraction of the L elements observed in a star was already present in the galactic gas at the birth of the star.

(1) The upper limit of interstellar $\mathrm{Be}, n(\mathrm{Be}) / n(\mathrm{H})<7 \times 10^{-11}$, is more than ten times smaller than the largest values observed in stars $\left(10^{-9}\right)$. It would seem improbable that $90 \%$ of the Be would be locked in grains. (Available upper limits on interstellar $\mathrm{Li}$ and $\mathrm{B}$ are too high to be of any interest. More sensitive determinations are needed.)

(2) In the star $T$ Tauri, the gas component with positive velocity has less than ten times the Li content of the star itself. This was considered so far as one of the strongest arguments in favor of the 'individual star' origin of Li. Herbig tells us that the matter may not be that simple (see the Discussion) since the Li-devoid gas shell appears to come from the star itself.

(3) Observations reported by Feast at this Conference suggest a regeneration of Li after a star has left the main-sequence.

(4) The largest ${ }^{7} \mathrm{Li} /{ }^{6} \mathrm{Li}$ ratios observed so far are approximately equal to the proton-induced spallation ratios $(\simeq 2 \cdot 5)$ reported by Dr. Gradsztajn. (The alteration mechanisms, to be discussed later, always destroy ${ }^{6} \mathrm{Li}$ faster than ${ }^{7} \mathrm{Li}$ (Figure 1).)

In the same figure, we see that the ratio of the destruction rates of $\mathrm{Li}$ and $\mathrm{Be}$ varies with proton energies, hence a similar argument cannot be made with observed $\mathrm{Li} / \mathrm{Be}$ ratios.

Other theories of $\mathrm{Li}, \mathrm{Be}, \mathrm{B}$ formation involve either a primordial origin (say from the Big Bang), or a gradual enrichment from Supernovae.

Recent work on the Big Bang (Wagoner et al., 1967) suggests a primordial yield

Perek (ed.), Highlights of Astronomy, 255-260. (C I.A.U. 


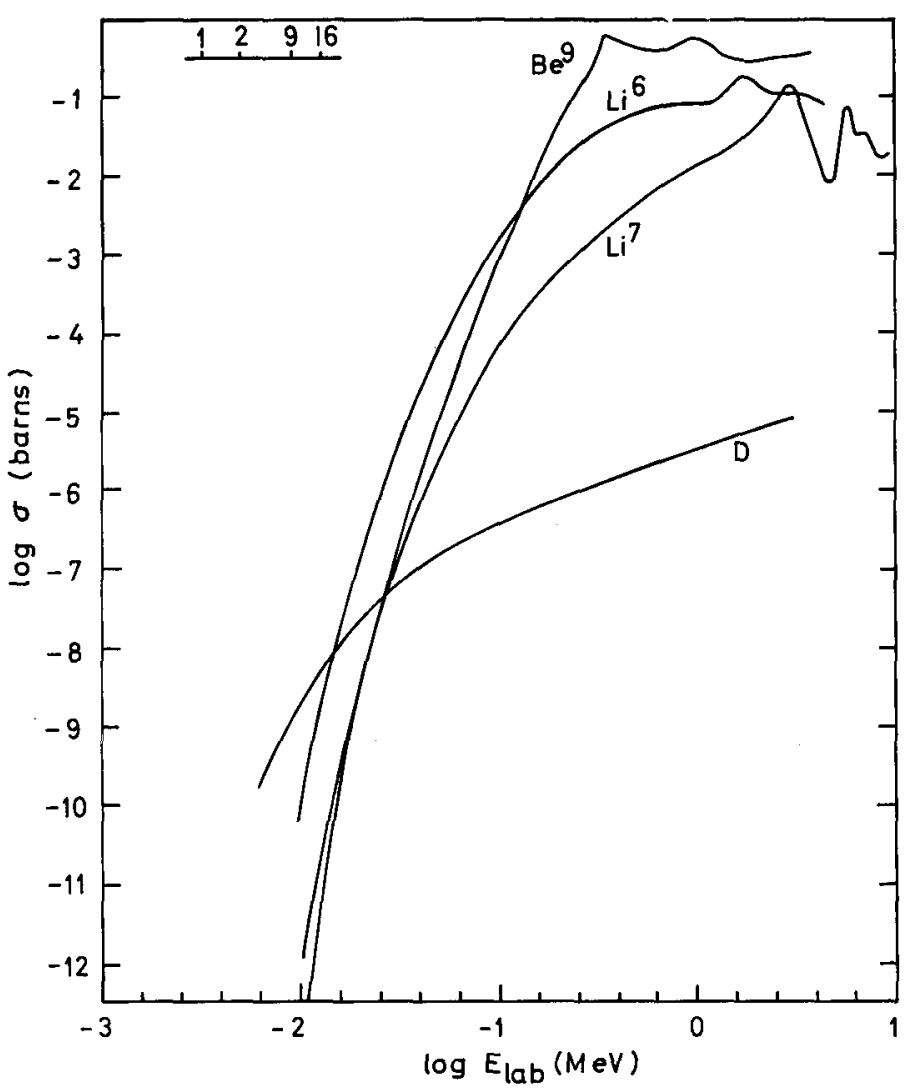

FIG. 1. Experimental cross-sections for the destruction of $\mathrm{D},{ }^{6} \mathrm{Li},{ }^{7} \mathrm{Li},{ }^{9} \mathrm{Be}$, as a function of the proton incident energies. Note the very small scale of the ordinates. The insert in the upper left shows the temperature in million degrees, for which the Gamow energies of Be are those of the abscissa. For instance, at $T_{6} \simeq 10$, the Gamow energy of Be(or Li) is approximately $10 \mathrm{keV}$.

of enough ${ }^{7} \mathrm{Li}$, but of not enough ${ }^{6} \mathrm{Li}$ and ${ }^{9} \mathrm{Be}$. The existence of many stars with ${ }^{7} \mathrm{Li} /{ }^{6} \mathrm{Li} \simeq 2$, hence shows, that Big Bang contribution is at best small.

Recent observations of $\mathrm{Be}$, reported by Conti at this Conference have important implications on this problem. When plotted against spectral class, the Be abundances are found to increase with decreasing surface temperature (hence deepening surface convective zone). As we shall argue later, Be atoms are most likely not destroyed in these stars. These observations may show a relationship between the intensity of the generation mechanism and the spectral class (thereby implying a mass effect, hence an 'individual star' origin). In view of our previous discussion, we are led to prefer this possibility.

Another possibility has been suggested by Conti (preprint): since late type stars are on the average older than early-type stars (the stars observed here are field stars), 
we may have here an age effect which could be explained in terms of a gradual destruction of the beryllium of the galactic gas (e.g. through stellar burning). This interpretation is made somewhat unlikely by the fact that several stars with no detectable $\mathrm{Be}$ (and also the solar system) do not seem to fit very well in this pattern. The question could be settled by a study of Be in various clusters.

At any rate, the implication of this observation is that the pattern of gradual enrichment of element abundances by nucleosynthesis in stars or supernovae does not apply to beryllium.

We now discuss briefly the importance of alphas and secondary neutron-induced reactions.

The contribution of alpha-induced spallation is probably small. The ratio of accelerated alphas to protons is generally expected to be small (and is observed to be small in the Sun and in the galactic cosmic rays). Furthermore, a systematic analysis of spallation rections (Audouze et al., 1967a) has shown that the relative yields of different isotopes is influenced mostly by the nuclear properties (binding energy, isotopic spin, density of levels) of the final product, so that we expect the cross-section ratio to be quite similar. Preliminary experimental results (Cuer preprint) corroborate this view.

Secondary neutrons will play no role if the mean proton energy in the flare is less than one hundred $\mathrm{MeV}$. At higher energies, and if the flare medium is dense enough ( $>10^{12}$ particles $\mathrm{cm}^{-3}$ ), the formation ratio of $\mathrm{Li} / \mathrm{Be}$ decreases slowly from about 20 to about 3 at one $\mathrm{GeV}$. (The ${ }^{7} \mathrm{Li} /{ }^{6} \mathrm{Li}$ ratio is insensitive to neutrons.) Hence, part, but not all of the variation of $\mathrm{Li} / \mathrm{Be}$ with spectral class could be explained by making the assumption that (1) neutrons do not decay in flight, and (2) the mean proton energy increases with decreasing surface temperature. We consider this hypothesis as unlikely, but we do not reject it at once.

B. How is the intensity of spallogeneration related to stellar evolution, and what is the influence of stellar mass, rotational velocities, and age?

The large abundances of Li observed in TTauri stars, and in particular in FU Orionis, suggest that the very early stages of stellar evolution witness a large part (if not most) of the electromagnetic activity of a star. If we are correct in interpreting the Be spectral class correlation as a mass effect, then we are prevented from pushing back the moment of irradiation too far in the past. In terms of proto-cluster evolution, the irradiation must clearly take place at a moment when the fractionation in stars is already well established, and each star must already know of what mass (hence of what spectral class) it is going to be.

From the data presented today, there seems to be a regenerescence of spallation reactions as the star leaves the M.S.: both the total $\mathrm{Li}$ and the ${ }^{7} \mathrm{Li} /{ }^{6} \mathrm{Li}$ ratio appear to increase before they decrease again as the star becomes a red giant. A coherent pattern emerges; spallation reactions are associated with major structural changes 
in the star (initial gravitational contraction; post M.S. contraction). These changes must bring important disturbances to the state of the atmosphere and, in turn, these disturbances must accelerate large particle fluxes, when coupled with the surface magnetic field and stellar rotation. In this sense, the problem of $\mathrm{Li}$ and $\mathrm{Be}$ is not a purely 'superficial' one anymore (in both senses of the word), but must be deeply related ro the problem of stellar evolution.

C. How and where were the spallogenic element abundances further altered? How is the alteration mechanism related to mass-rotational velocity, age, surface properties, etc?

Herbig has pointed out that the total stellar $\mathrm{Li}$ abundance appears to decrease with stellar age, with an average lifetime of $10^{9}$ years. A similar conclusion can be drawn from a comparison between the Hyades and the Pleiades. The fact that varying $\mathrm{Li} / \mathrm{Be}$ (as mentioned before, only part of the variation could come from secondary neutron-induced reactions) and ${ }^{7} \mathrm{Li} /{ }^{6} \mathrm{Li}$ ratios are observed shows that the depletion must be, for an important part, due to nuclear destruction (and not only to dilution). In stellar surfaces (and interiors), spallogenic element abundances can only be altered by proton-capture reactions (alpha-capture probabilities are reduced by Coulomb repulsion, neutron-capture occurs predominantly on hydrogen and has no effect on $\mathrm{Li}$ or $\mathrm{Be}$ ). The fact that all ${ }^{7} \mathrm{Li} /{ }^{6} \mathrm{Li}$ ratios observed are larger than the proton formation ratio is coherent with this affirmation; as seen from Figure 1, the capture cross-section of ${ }^{6} \mathrm{Li}$ is at all energies considerably larger than the ${ }^{7} \mathrm{Li}$ capture cross-section.

It has been assumed so far that $\mathrm{Li}$ depletion occurs mostly in stellar surface convective zone (where the temperatures do not exceed a few million degrees, hence Gamov energies do not exceed 5 or $10 \mathrm{keV}$ ). We must, however, consider the possibility that $\mathrm{Li}$ is destroyed by the spallogenerating proton flux itself. The abundance equation of an element $\mathrm{L}$ must indeed be written as:

$$
\begin{aligned}
\frac{\mathrm{d} n_{\mathrm{L}}}{\mathrm{d} t} & ={ }^{n}{ }_{\mathrm{CNO}} \int_{Q}^{\infty} \Phi(E p) \sigma_{s p}(\mathrm{CNO} \rightarrow \mathrm{L}) \mathrm{d} E \\
& -n_{\mathrm{L}} \int_{0}^{\infty} \Phi(E p) \sigma_{d e s}(\mathrm{~L}) \mathrm{d} E-n_{\mathrm{L}} \int_{0}^{\infty} n(E p) \sigma_{d e s}(\mathrm{~L}) v \mathrm{~d} E .
\end{aligned}
$$

We recognize first the formation term, proportional to the high-energy part of the proton flux $\Phi(E p)$; the surface destruction term and the convective zone destruction term, proportional to the proton density $n(E p)$, (with a M.B. energy distribution). The main difference between these last two terms is that $\Phi(E p)$ has a higher mean energy, but a much lower density than $n(E p)$.

The relative importance of the two destruction terms is closely related to the location of the most probable energy at which stellar lithium is destroyed. The very low 
$n(\mathrm{Li}) / n(\mathrm{CNO})$ observed in stars $\left(\leqslant 10^{-5}\right)$ rules out the energy range over a few tens of $\mathrm{MeV}$; important destruction in this range would imply important destruction of $\mathrm{CNO}$ and larger $n(\mathrm{Li}) / n(\mathrm{CNO})$ ratios.

A more probable region is the $\mathrm{MeV}$ and sub-MeV region; if the proton spectrum keeps on increasing with decreasing energy (as suggested by the solar cosmic-ray spectrum), we may expect important effects from the first term, at a 'Gamow' energy defined by the maximum of the integrand. Even for extremely steep spectrum, the Gamow energy cannot be below $25 \mathrm{keV}$.

In Figure 1, we note that at $E p<25 \mathrm{keV}, \sigma(\mathrm{Be})<\left({ }^{7} \mathrm{Li}\right)$ and at $E p>25 \mathrm{keV}, \sigma(\mathrm{Be})>\left({ }^{7} \mathrm{Li}\right)$.

This crossing of the cross-sections around $25 \mathrm{keV}$ is most useful, since it permits a clear separation between surface destruction $\left(E_{G} \geqslant 25 \mathrm{keV}\right)$, and convective-zone destruction $\left(E_{G} \leqslant 25 \mathrm{keV}\right)$. The presence of ${ }^{6} \mathrm{Li}$ will not perturb this separation very much.

Conti and Danziger have measured $\mathrm{Li} / \mathrm{Be}$ ratios in a number of stars, and have shown that this ratio is always smaller than the formation ratio $(\simeq 20)$. This observation shows that the most probable destruction energy is below $25 \mathrm{keV}$, and that stellar $\mathrm{Li}$ destruction occurs mostly in convective zones. This conclusion is of course strengthened by the correlation between $\mathrm{Li} / \mathrm{Be}$ ratio and spectral classes (such a correlation would not a priori be expected in the case of surface destruction).

D. What can we say about energies involved in the process, and in particular about the efficiency of the accelerating mechanism?

If a star contains $n_{\mathrm{L}}$ atoms of a spallogenic element, the energy lost by the star in spallation is simply $E_{\mathrm{L}}^{\mathrm{I}}=n_{\mathrm{L}} Q_{\mathrm{L}} \simeq 10^{43}$ ergs in typical stars. (The notation $E^{\mathrm{I}}$ refers to the fact that this mechanism belongs to the realm of the first law of thermodynamics.) This loss is small and unimportant.

On the other hand, the amount of energy which must have been used in acceleration is given by:

$$
E_{\mathrm{L}}^{\mathrm{II}}=n_{\mathrm{L}}\left\langle\frac{\varepsilon n_{\mathrm{H}}}{n_{\mathrm{CNO}} \sigma(\mathrm{CNO} \rightarrow \mathrm{L})}\right\rangle \simeq 10^{48} \mathrm{ergs},
$$

where $\varepsilon$ is the stopping power of the gas, and the mean value is taken over the proton spectrum. The notation $E^{\mathrm{II}}$ (second law of thermodynamics) refers to the fact that this energy is not lost by the star, but largely recuperated through the electronic collisions. This energy is significant in that it characterizes the efficiency of the accelerating mechanism. Its bearing on the theory of stellar evolution has been briefly discussed by Audouze et al. (1967b).

\section{References}

Audouze, J., Epherre, M., Reeves, H. (1967a)

Audouze, J., Gradsztajn, E., Reeves, H. (1967b)

41, p. 299.

Wagoner, R.V., Fowler, W.A., Hoyle, F. (1967)
Nucl. Phys., A97, 144.

Les Congrès et Colloques de l'Université de Liège, Astrophys. J., 148, 3. 


\section{DISCUSSION}

Fowler: Did you notice that several stars with depleted $\mathrm{Li}$ have ${ }^{6} \mathrm{Li} /{ }^{7} \mathrm{Li} \simeq \frac{1}{2}$, which is the unmodified spallation value? I have suggested that we are seeing $\mathrm{Li}$, which has not been involved in the convection. Do you have an alternative explanation?

Reeves: I fully agree with you. If we try to imagine what actually did happen, and what information we can extract from the observed ${ }^{6} \mathrm{Li} /{ }^{7} \mathrm{Li} / \mathrm{Be}$ value, then we are led to several possibilities. These possibilities are based on the idea that the spallogenic elements observed are really the sum of two contributions: an 'old' one, in which all $\mathrm{Li}$ has been destroyed, but some Be has survived, and a 'new' one, in which all three isotopes are present in their formation ratios. With the formation ratios quoted by Dr. Gradsztajn, it is of course easy to evaluate the fractional importance of each contribution. Let me mention three possibilities.

(I) Some Be would have been present in the original stellar gas. According to our previous discussion, this is unlikely, but can certainly not be ruled out at this time.

(II) The first contribution may have been produced during the very early stages, followed by a thorough destruction of $\mathrm{Li}$ during the Hayashi convective period, and followed by a second irradiation somewhat later, e.g. at the time when the star settles on the M.S. The variation in the ${ }^{7} \mathrm{Li} /{ }^{6} \mathrm{Li}$ ratio would then show the extent of ${ }^{6} \mathrm{Li}$ burning since the star is on the M.S.

(III) Let us suppose that some spallogeneration is still going on at the surface of these stars. If the average diffusion time ' $t \mathrm{~d}$ ' for the spallogenic elements to sink from the optical region into the convective zone is not too short or too long, then we could observe in the spectrum both a 'recent' contribution of elements which have not yet sunk in the convective zone, and an 'old' contribution from all elements previously generated and diluted through the convective zone (assumed to be hot enough to burn $\mathrm{Li}$, but not $\mathrm{Be}$ ). The critical time-scale for the diffusion time to be such that both contributions are comparable is $t_{\mathrm{d}} \simeq x T$, where $x$ is the dilution factor between the irradiated mass and the convective mass, and $T$ is related to the age of the star. Typical values of $x$ range from $10^{-7}$ to $10^{-9}$, so that the required $t_{\mathrm{d}}$ would vary from a small fraction of a year to several years, according to the star under consideration. I have questioned several experts on atmospheres. None of them dismissed such periods as unlikely. As mentioned before, in such cases, ${ }^{7} \mathrm{Be}$ and ${ }^{10} \mathrm{Be}$ could well be observed. Observers should be on the look.

Masevic: If all the $\mathrm{Li}$ is destroyed at the bottom of the outer convective zone, the theoretically expected abundances will include all uncertainties of the theory of the convective zone (mixing lengths, etc.). That will make the relation between the observed $\mathrm{Li}$ abundances and the evolution still more difficult to investigate, at least for M.S. stars.

Reeves: According to Miss Merchant, all observed Li/Be ratios are smaller than the proton-induced formation ratio, hence I am afraid that we have to face the difficult situation that you are describing.

Herbig (after an unreported remark of Dr. Danziger): I believe that the Bonsack-Greenstein observation (i.e. that in $\mathrm{T}$ Tauri there is no $\mathrm{Li}$ line in the displaced shell spectrum) does not tell us anything about pre-stellar material. The shell lines are violet-displaced, so the material is rising from the star, and so this is material that has already been processed. Of course, why the $\mathrm{Li}$ is not seen in this rising gas is another problem. 